\title{
Korean Brain Aging Study for the Early Diagnosis and Prediction of Alzheimer's Disease: Methodology and Baseline Sample Characteristics
}

\author{
Min Soo Byun ${ }^{1}$, Dahyun Yi', Jun Ho Lee ${ }^{2}$, Young Min Choe ${ }^{3}$, Bo Kyung Sohn ${ }^{4}$, Jun-Young Lee ${ }^{5,6}$, \\ Hyo Jung Choi ${ }^{5}$, Hyewon Baek ${ }^{7}$, Yu Kyeong Kim ${ }^{8}$, Yun-Sang Lee ${ }^{9}$, Chul-Ho Sohn ${ }^{10}$, Inhee Mook-Jung ${ }^{11,12}$, \\ Murim Choi ${ }^{12}$, Yu Jin Lee ${ }^{2,6}$, Dong Woo Lee ${ }^{4}$, Seung-Ho Ryu ${ }^{13}$, Shin Gyeom Kim ${ }^{14}$, Jee Wook Kim ${ }^{15}$, \\ Jong Inn $\mathrm{Woo}^{6}$, Dong Young Lee $\mathrm{e}^{1,2,6} \bowtie$, and for the KBASE Research Group* \\ ${ }^{1}$ Institute of Human Behavioral Medicine, Medical Research Center Seoul National University, Seoul, Republic of Korea \\ ${ }^{2}$ Department of Neuropsychiatry, Seoul National University Hospital, Seoul, Republic of Korea \\ ${ }^{3}$ Department of Neuropsychiatry, University of Ulsan College of Medicine, Ulsan University Hospital, Ulsan, Republic of Korea \\ ${ }^{4}$ Department of Psychiatry, Sanggye Paik Hospital, Inje University College of Medicine, Seoul, Republic of Korea \\ ${ }^{5}$ Department of Neuropsychiatry, SMG-SNU Boramae Medical Center, Seoul, Republic of Korea \\ ${ }^{6}$ Department of Psychiatry, Seoul National University College of Medicine, Seoul, Republic of Korea \\ ${ }^{7}$ Department of Neuropsychiatry, Kyunggi Provincial Hospital for the Elderly, Yongin, Republic of Korea \\ ${ }^{8}$ Department of Nuclear Medicine, SMG-SNU Boramae Medical Center, Seoul, Republic of Korea \\ ${ }^{9}$ Department of Nuclear Medicine, Seoul National University College of Medicine, Seoul, Republic of Korea \\ ${ }^{10}$ Department of Radiology, Seoul National University College of Medicine, Seoul National University Hospital, Seoul, Republic of Korea \\ ${ }^{11}$ Department of Biochemistry, Seoul National University College of Medicine, Seoul, Republic of Korea \\ ${ }^{12}$ Department of Biomedical Science, Seoul National University College of Medicine, Seoul, Republic of Korea \\ ${ }^{13}$ Department of Psychiatry, School of Medicine, Konkuk University, Konkuk University Medical Center, Seoul, Republic of Korea \\ ${ }^{14}$ Department of Neuropsychiatry, Soonchunhyang University Bucheon Hospital, Bucheon, Republic of Korea \\ ${ }^{15}$ Department of Neuropsychiatry, Hallym University Dongtan Sacred Heart Hospital, Hwaseong, Republic of Korea
}

Objective The Korean Brain Aging Study for the Early Diagnosis and Prediction of Alzheimer's disease (KBASE) aimed to recruit 650 individuals, aged from 20 to 90 years, to search for new biomarkers of Alzheimer's disease (AD) and to investigate how multi-faceted lifetime experiences and bodily changes contribute to the brain changes or brain pathologies related to the AD process.

Methods All participants received comprehensive clinical and neuropsychological evaluations, multi-modal brain imaging, including magnetic resonance imaging, magnetic resonance angiography, $\left[{ }^{11} \mathrm{C}\right]$ Pittsburgh compound B-positron emission tomography (PET), and $\left[{ }^{18} \mathrm{~F}\right]$ fluorodeoxyglucose-PET, blood and genetic marker analyses at baseline, and a subset of participants underwent actigraph monitoring and completed a sleep diary. Participants are to be followed annually with clinical and neuropsychological assessments, and biannually with the full KBASE assessment, including neuroimaging and laboratory tests.

Results As of March 2017, in total, 758 individuals had volunteered for this study. Among them, in total, 591 participants-291 cognitively normal $(\mathrm{CN})$ old-aged individuals, $74 \mathrm{CN}$ young- and middle-aged individuals, 139 individuals with mild cognitive impairment (MCI), and 87 individuals with $\mathrm{AD}$ dementia (ADD)-were enrolled at baseline, after excluding 162 individuals. A subset of participants $(\mathrm{n}=275)$ underwent actigraph monitoring.

Conclusion The KBASE cohort is a prospective, longitudinal cohort study that recruited participants with a wide age range and a wide distribution of cognitive status (CN, MCI, and ADD) and it has several strengths in its design and methodologies. Details of the recruitment, study methodology, and baseline sample characteristics are described in this paper. Psychiatry Investig 2017;14(6):851-863

Key Words Alzheimer's disease, Early diagnosis, Prediction, Biomarker, Prospective cohort study.

\footnotetext{
Received: April 17, 2017 Revised: June 27, 2017 Accepted: July 8, 2017 Available online: October 12, 2017

$\triangle$ Correspondence: Dong Young Lee, $\mathrm{MD}, \mathrm{PhD}$

Department of Neuropsychiatry, Seoul National University Hospital, Department of Psychiatry, Seoul National University College of Medicine, 101 Daehak-ro, Jongno-gu, Seoul 03080, Republic of Korea

Tel: +82-2-2072-2205, Fax: +82-2-744-7241, E-mail: selfpsy@snu.ac.kr

*Information on the KBASE Research Group is provided in the Supplementary Materials 2 (in the online-only Data Supplement).

(a) This is an Open Access article distributed under the terms of the Creative Commons Attribution Non-Commercial License (http://creativecommons.org/licenses/by-nc/4.0) which permits unrestricted non-commercial use, distribution, and reproduction in any medium, provided the original work is properly cited.
} 


\section{INTRODUCTION}

Alzheimer's disease $(\mathrm{AD})$ is the most common cause of dementia. ${ }^{1,2}$ Because older age itself is a primary risk factor for $\mathrm{AD}$, the number of people living with $\mathrm{AD}$ is increasing rapidly and is projected to reach over 100 millions worldwide by 2050, resulting in tremendous social and economic impacts globally. ${ }^{2-4}$ Development of effective therapeutic interventions that can delay or prevent disease progression has become a public health priority, notably since previous studies postulated that the prevalence and economic burden of $\mathrm{AD}$ could decrease by almost $50 \%$ in 2050 if the onset of $\mathrm{AD}$ is delayed by 5 years in 2015.5

Nevertheless, the underlying etiologies and pathophysiology of $\mathrm{AD}$ are not yet fully understood and there is currently no effective treatment that can cure $\mathrm{AD}$. Moreover, recent clinical trials using anti-amyloid therapies for mild-to-moderate $\mathrm{AD}$ dementia (ADD) patients that were expected to modify the disease-course of AD failed to meet the primary endpoints. ${ }^{7-9}$ Regarding the disappointing outcome of these trials, researchers pointed out that the timing of intervention may have been one of the possible reasons; for patients with dementia stage of $\mathrm{AD}$, it may be too late for disease-course modification because of the irreversible neuronal loss that would mitigate any effect of therapy. ${ }^{8,10}$ Thus, early detection and intervention before neuronal loss are likely to be important for maximizing the beneficial effect of new therapies. Furthermore, the targets for developing disease-modifying agents are shifting from patients with ADD to individuals in the predementia phase and even in the asymptomatic stage of $\mathrm{AD} .{ }^{11}$ In this context, development and validation of $\mathrm{AD}$ biomarkers for the early detection and prediction of $\mathrm{AD}$ with high accuracy have become important research goals. ${ }^{12,13}$

Several neuroimaging techniques including positron emission tomography (PET) and magnetic resonance imaging (MRI) and cerebrospinal fluid (CSF) biomarkers that reflect $\beta$-amyloid $(\mathrm{A} \beta)$ deposition and $\mathrm{AD}$-specific neurodegeneration are currently available. ${ }^{12-14}$ Although these biomarkers have markedly advanced clinical research and practice regarding $\mathrm{AD}$ and have been incorporated into recent diagnostic criteria for $\mathrm{AD}$, as recommended by the National Institute of Aging and Alzheimer's Association (NIA-AA) ${ }^{15}$ and International Working Group, ${ }^{16}$ these biomarkers have limitations for broader applications. In case of CSF biomarkers, lumbar puncture for collecting CSF samples requires invasive, labor-intensive procedures, and measurement variability due to lack of standardization and poor inter-institution reliability is still problematic. ${ }^{13,17,18}$ While the neuroimaging biomarkers have strengths in being non-invasiveness and having good face validity, they still have limitations; most notably, not only the biomarkers using PET are expensive and are not commonly available except at tertiary hospitals, but they also come with the risk of radiation exposure for serial or repeated scans. ${ }^{17,19,20}$ Additionally, clinical interpretations of neuroimaging-based neuronal injury biomarkers are mostly dependent on visual qualitative reading because of limited normative information for quantitative reading, particularly in non-Caucasian population. ${ }^{21}$ Thus, less invasive, less expensive, more readily available "new" biomarkers that are highly accurate and reliable for AD are needed, especially given increasing need for biomarkers that can be used on the front line in widespread screening in clinical practice and for the recruitment of candidates for clinical trials, as well as for repeated use for monitoring disease progression and treatment efficacy. For example, blood-based biomarkers that can be assessed with simple venipuncture will be candidates if they reflect the underlying AD pathology. ${ }^{17}$ Several previous studies have attempted to discover novel biomarkers for $\mathrm{AD}$ from blood-derived specimens (i.e., plasma, DNA, etc.) along with advancements in analytical methods such as proteomic and genetic analyses. ${ }^{17,22-25}$ The previously reported candidates, however, have limited utilization due to variable accuracy and low reproducibility stemming from two factors: 1) for several studies, samples were collected based on clinical diagnosis criteria rather than presence of in vivo $\mathrm{AD}$ pathologies and 2) sample sizes were relatively small for some studies. Therefore, further large-scale, well-characterized cohort studies are necessary to discover and validate biomarkers including blood-based ones that can reflect in vivo brain $\mathrm{AD}$ pathologies.

Various risk or protective factors for dementia syndrome or cognitive impairment have been suggested by numerous epidemiological studies. ${ }^{1,26}$ However, we still do not know much about the mechanisms through which various lifetime experiences such as physical and mental activity, social relationships, stress experiences, personality, diet and nutrition, and sleep, and many aging-related physiological or pathological changes in the human body, such as vascular status, hormonal changes, glucose or lipid metabolism, and body mass index (BMI) contribute to AD-related brain changes. ${ }^{1,3}$ Thus, the mechanisms that link these factors to specific in vivo $\mathrm{AD}$ pathologies should be clarified for the development of more specific preventative or disease-modifying interventions for AD. More specifically, it is necessary to clarify whether a risk or protective factor contributes directly to $A \beta$ accumulation or whether it aggravates neuronal injury through an $A \beta$ independent mechanism in the living human brain. Additionally, to adopt modifiable risk or protective factors into treatment and prevention, it is important to know the critical lifetime period and duration of exposure when a certain risk or protective factor has a significant impact on $\mathrm{AD}$-related brain chang- 
es and to understand its interaction with cognitive or neural reserves and genetic predisposition. ${ }^{1,27}$

Given this background, the Korean Brain Aging Study for the Early Diagnosis and Prediction of Alzheimer's disease (KBASE) cohort, a prospective observational cohort which started in 2014, aimed to develop new biomarkers, particularly blood biomarkers, for the early detection of $\mathrm{AD}$ processes or pathologies. A multidisciplinary team participated in the search for biomarkers by applying advanced imaging analysis, biochemical, proteomics, genetics, and big data analysis techniques to the cohort data. The study also aimed to investigate how multi-faceted lifetime experiences and bodily changes contribute to the brain changes or brain pathologies related to $\mathrm{AD}$ process. We hypothesized that investigating the association of lifetime experiences or bodily changes with in vivo $\mathrm{AD}$ pathologies measured by $\mathrm{AD}$ biomarkers in individuals with varied ages and cognitive status could reveal modifiable contributors to $\mathrm{AD}$ pathologies. This paper reports the study methodology and the baseline demographic, clinical and neuropsychological characteristics of participants in the KBASE cohort.

\section{METHODS}

\section{Recruitment of participants}

The study aimed to recruit 650 individuals overall [70 cognitively normal $(\mathrm{CN})$ young and middle-aged adults (CN-ym), 350 CN old-aged adults (CN-old), 140 individuals with mild cognitive impairment (MCI) and 90 individuals with ADD]. First, individuals who met the following criteria were included as the CN-ym group: 1) age 20-54 years (inclusive), 2) Clinical Dementia Rating (CDR) score of 0 , and 3 ) no diagnosis of $\mathrm{MCI}$ or dementia. For CN-old group, inclusion criteria were 1) age 55-90 years (inclusive), 2) CDR score of 0 , and 3) no diagnosis of MCI or dementia. For the MCI group, individuals [age 55-90 years (inclusive)] who met the following inclusion criteria based on core clinical criteria for diagnosis of MCI according to the recommendations of the NIA-AA guidelines, ${ }^{28}$ were included: 1) memory complaint corroborated by self, an informant, or clinician, 2) objective memory impairment for age, education, and gender, 3) largely intact functional activities, and 4) not demented. All MCI individuals had a global CDR score of 0.5. In terms of criterion 2) for MCI, all MCI participants had a performance score at least $1.0 \mathrm{SD}$ below the respective age, education, and gender-specific mean for at least one of the four episodic memory tests included in the Korean version of Consortium to Establish a Registry for Alzheimer's Disease (CERAD-K) neuropsychological battery (Word List Memory, Word List Recall, Word List Recognition and Constructional Recall test). ${ }^{29}$ Finally, participants with ADD [age
55-90 years (inclusive)] who met the following inclusion criteria were recruited: (a) criteria for dementia in accordance with the Diagnostic and Statistical Manual of Mental Disorders 4th Edition (DSM-IV-TR), ${ }^{30}$ (b) the criteria for probable ADD in accordance with the NIA-AA guidelines, ${ }^{15}$ and (c) CDR score of 0.5 or 1 . Exclusion criteria for all participants include: 1) presence of major psychiatric illness (e.g., schizophrenia, bipolar disorder, major depressive disorder, alcohol/substance abuse or dependence, delirium), 2) significant neurological or medical condition or comorbidities that could affect mental functioning, 3) contraindications for MRI scan (e.g. pacemaker, claustrophobia), 4) illiteracy, 5) presence of significant visual/hearing difficulty, severe communication or behavioral problems that would make a clinical examination or brain scan difficult, 6) taking an investigational drug, and 7) in pregnancy or breastfeeding.

Participants were recruited through four recruitment sites (two university hospitals and two public centers for dementia prevention and management) around Seoul, South Korea. In addition, volunteers from the community were recruited through advertisements at an online homepage, posters, and brochures provided at main recruitment sites and word of mouth (recommended by other participants, family members, friends, or acquaintances). In the MCI and ADD groups, potentially eligible individuals who visited memory clinics at two university hospitals [Seoul National University Hospital (SNUH) and Seoul National University-Seoul Metropolitan Government (SNU-SMG) Boramae Medical Center] or participated in a dementia screening program at two public centers for dementia prevention and management were informed about study participation and those who volunteered were invited for an assessment of eligibility.

\section{Ethics approval}

This study protocol was approved by the Institutional Review Boards of SNUH (C-1401-027-547) and SNU-SMG Boramae Medical Center (26-2015-60), Seoul, South Korea, and was conducted in accordance with the recommendations of the current version of the Declaration of Helsinki. The subjects or their legal representatives gave written informed consent.

\section{KBASE baseline assessment protocol}

At baseline, all participants underwent comprehensive clinical and neuropsychological assessments, multi-modal neuroimaging examinations including $\left[{ }^{11} \mathrm{C}\right]$ Pittsburgh compound $\mathrm{B}$ (PiB)-PET, $\left[{ }^{18} \mathrm{~F}\right]$ fluorodeoxyglucose (FDG)-PET, MRI and magnetic resonance angiography (MRA), and comprehensive laboratory blood tests. Clinical and neuropsychological assessments were done at SNUH and SMG-SNU Boramae Medical Center, and other baseline assessment including neuroimaging 
and blood sampling were done at a single center (SNUH).

\section{Clinical assessment}

All participants were administered standardized clinical assessments by trained board-certified psychiatrists based on the KBASE clinical assessment protocol which incorporated the CERAD-K clinical assessment ${ }^{31,32}$ at the time of recruitment. Reliable informants were also interviewed and medical records were reviewed for more accurate information if available. Global and each domain CDR score ${ }^{33}$ were rated for all participants and CDR-Sum-of-Box (CDR-SB) were calculated as a sum of the CDR score of all domains. The Global Deterioration Scale (GDS) was also administered for evaluation of dementia severity. The Blessed Dementia Scale-Activity of Daily Living (BDS-ADL) ${ }^{32,34}$ was administered to measure abilities in ADL. Physical and neurological examinations were also performed by clinicians.

The KBASE clinical assessment protocol incorporated a standardized interview form based on the Structured Clinical Interview for DSM-IV (SCID-IV) ${ }^{35}$ that is required for the diagnosis of present and past major depressive episodes. Additionally, the severity of depressive symptoms at the time of the examination was measured using the 17-item Hamilton Rating Scale for Depression, ${ }^{36}$ the 30 -item Korean version of the Geriatric Depression Scale (GDS-KR), ${ }^{37,38}$ and the Center for Epidemiologic Studies Depression scale (CES-D). ${ }^{39}$ The State-Trait Anxiety Inventory (STAI) ${ }^{40}$ was administered to all participants.

To evaluate subjective memory complaints, the Subjective Memory Complaints Questionnaire (SMCQ) ${ }^{41}$ was completed by all participants. The Seoul Informant Report Questionnaire for Dementia (SIRQD) ${ }^{42}$ was also completed by reliable informants.

Current and past medical comorbidities, family history (FH) of dementia, use of medication, nutraceuticals, smoking, alcohol, and coffee intake were assessed based on systematic interviews with participants and their informants by trained nurses. The presence or absence of vascular risk factors (VRFs), including hypertension, diabetes mellitus, coronary heart disease, hyperlipidemia, stroke, and transient ischemic attack (TIA) were also identified in the same manner and VRF score (VRS) was calculated as the number of the VRFs present and reported as a percentage. ${ }^{43}$ Medications were also reviewed based on prescriptions provided by participants and medical records. Height, weight, BMI and other anthropometric variables (i.e., circumferences of waist, hip, head, neck, upper arm, etc.) were measured. Systolic/diastolic blood pressure (SBP/ DBP) was measured in supine and erect positions by trained nurses using sphygmomanometer. Additionally, ankle brachial pressure index (ABI) was calculated based on the average
$\mathrm{BP}$ in the right arm and bilateral ankle (posterior tibial artery) measured with a hand-held Doppler device. ${ }^{44}$

\section{Neuropsychological assessment}

All subjects were given a comprehensive neuropsychological assessment battery, administered by clinical neuropsychologists or trained psychometrists, according to a standardized protocol incorporating the CERAD-K neuropsychological battery. ${ }^{29}$ The selected tests included in the battery are recognized internationally and are used widely to cover the main cognitive domains that are affected by $\mathrm{AD}$ and other dementias. The domains were attention, processing speed, verbal and nonverbal memory, visuospatial functioning, language, and executive functioning. The full battery consisted of the Mini-Mental State Examination in the Korean version of the CERAD assessment packet (MMSE-KC), ${ }^{29} \mathrm{CERAD-K}$ verbal memory tests, ${ }^{29}$ including Word List Memory, Word List Delayed Recall, Word List Recognition, CERAD-K Constructional Praxis, ${ }^{29}$ and CERAD-K nonverbal memory delayed recall, ${ }^{29}$ the Trail Making Test A and $\mathrm{B},{ }^{45}$ and the Stroop Test (Korean Golden version), ${ }^{46}$ Verbal Fluency Tasks (both semantic ${ }^{29}$ and phonemic ${ }^{47}$ ), CERAD-K confrontational naming test (Modified Korean version of the Boston Naming Test), ${ }^{29}$ Wechsler Adult Intelligence Scale-revised edition Korean version (WAIS-R-K) Digit Span (forward and backward), ${ }^{48,49}$ Wechsler Memory Scale-Fourth edition Korean version (WMS-IV-K) Logical Memory I, II and Recognition (both stories), ${ }^{50}$ Rey Complex Figure Test (RCFT), ${ }^{51}$ and the Wechsler Adult Intelligence Scale-Fourth edition Korean version (WAIS-IV-K) Block Design. ${ }^{52}$ The length of the full assessment was $-2 \mathrm{~h}$. All participants were also administered the Korean Adult Reading Test (KART) ${ }^{53}$ to estimate premorbid functioning.

\section{Consensus diagnostic conferences}

Allocation of individuals to one of the four diagnostic groups and exclusion of ineligible individuals were undertaken by a clinical review panel chaired by DYL at a weekly clinical review panel meeting. This panel consisted of several boardcertified psychiatrists, clinical neuropsychologists, and psychometrists who trained for research in geriatric psychiatry and dementia.

\section{Blood sampling and laboratory assessments}

Overnight fasting blood samples $(70 \mathrm{~mL})$ were collected in the morning by venipuncture and collected in K2 EDTA, SST, trace element serum tubes and PAXgene Blood RNA tubes. Baseline routine blood laboratory tests included complete blood cell count, erythrocyte sedimentation rate, BUN/creatinine, albumin, liver function tests, fasting serum glucose, 
glycated hemoglobin (HbAlc), serum lipids, homocysteine, thyroid function test, sex hormones, insulin, cortisol, zinc, copper, ferritin/transferrin/iron, and ceruloplasmin. Genomic DNA was extracted from whole blood and apolipoprotein $\mathrm{E}$ (APOE) genotyping was performed as described previously. ${ }^{54}$ APOE $\varepsilon 4$ carrier status was coded if at least one $\varepsilon 4$ allele was present.

The remaining blood samples were stabilized and centrifuged (700 g, $5 \mathrm{~min}$, room temperature) to obtain plasma and serum supernatants, and buffy coat. To obtain samples with high purity, the plasma and serum supernatants were further centrifuged under the same conditions, and the collected pure plasma and serum supernatants were aliquoted and immediately stored at $-80^{\circ} \mathrm{C}$. Buffy coat, DNA, and RNA specimens were also stored at $-80^{\circ} \mathrm{C}$ for future analyses.

To find candidates for novel blood-based biomarkers of $\mathrm{AD}$, plasma proteomics analyses and biochemical validation using the enzyme-linked immunosorbent assay and X-MAP technology are ongoing. Additionally, genetic analyses using next generation sequencing technologies including whole genome sequencing as well as targeted gene panel analyses are underway.

\section{Neuroimaging}

Participants underwent two-sessions of PET-MR scanning to obtain multi-modal imaging including $\left[{ }^{11} \mathrm{C}\right] \mathrm{PiB}-\mathrm{PET}$, $\left[{ }^{18} \mathrm{~F}\right]$ FDG-PET, MRI, and MRA using a 3.0T Biograph mMR (PETMR) scanner (Siemens, Washington, DC, USA) according to the manufacturer's approved guidelines. Detailed information on image preprocessing procedures will be described in more specialized papers.

\section{$\left[{ }^{11} \mathrm{C}\right]$ PiB-PET}

Participants underwent simultaneous three-dimensional (3D) $\left[{ }^{11} \mathrm{C}\right] \mathrm{PiB}-\mathrm{PET}$ and 3D T1-weighted MRI using the 3.0T PET-MR scanner. After intravenous administration of $555 \mathrm{MBq}$ of $\left[{ }^{11} \mathrm{C}\right] \mathrm{PiB}$ (range, 450-610 MBq), a 30-min emission scan was obtained 40 min after injection. The PiB-PET data collected in list mode were processed for routine corrections such as uniformity, UTE-based attenuation, and decay corrections, and were reconstructed into a $256 \times 256$ image matrix using iterative methods (6 iterations with 21 subsets).

\section{$\left[{ }^{18}\right.$ F]FDG-PET}

The participants fasted for at least $6 \mathrm{~h}$ and rested in a waiting room for $40 \mathrm{~min}$ prior to the scans after intravenous administration of $0.1 \mathrm{mCi} / \mathrm{Kg}$ of $\left[{ }^{18} \mathrm{~F}\right] \mathrm{FDG}$ radioligands. The PET data collected in list mode ( $5 \min \times 4$ frames) were processed for routine corrections such as uniformity, UTE-based attenuation, and decay corrections. After inspecting the data for any significant head movements, we reconstructed them into a 20-min summed image using iterative methods (6 iterations with 21 subsets).

\section{MRI and MRA}

MRI and MRA scan data were obtained from all participants using the 3.0T PET-MR scanner. Both structural MRI, including 3D T1, T2, fluid-attenuated inversion recovery (FLAIR), diffusion tensor imaging (DTI), and susceptibility weighted imaging (SWI) sequences and functional MRI (fMRI), including resting-state fMRI and arterial spin labeling (ASL), were obtained. All MR images were reviewed by neuroradiologists.

\section{Lifetime experience \& personality assessments}

All participants in the KBASE cohort were comprehensively evaluated for multi-faceted lifetime experiences and personality traits. More detailed and specific demographic variables including current and past occupational attainments, socioeconomic status, residence, information on family members, and religious activity were obtained from semi-structured interviews. Several self-reported and/or informant-reported questionnaires to assess the lifetime cognitive and physical activity, lifetime history of stressful events, diet and nutrition, lifetime changes in body weights, personality traits, resilience, social support, interpersonal reactivity, religiosity/ spirituality, and anosognosia were administered to participants and/or informants. Instruments or questionnaires used in the KBASE are described in the Supplementary Materials 1 (in the online-only Data Supplement).

\section{Assessment of sleep-circadian rhythm}

All subjects completed subjective sleep-related questionnaires including the Pittsburgh Sleep Quality Index, ${ }^{55}$ The REM Sleep Behavior Disorder Questionnaire, ${ }^{56}$ STOP questionnaire, ${ }^{57}$ Cambridge-Hopkins questionnaire for restless legs syndrome (CH-RLSq) ${ }^{58}$ and Morningness-eveningness questionnaireKorean version (MEQ-K). ${ }^{59}$ Subjectively reported lifetime sleep habits including average sleep duration and sleep quality from adolescent to late-life were also collected through interviews with participants and informants by trained nurses. To objectively measure sleep-circadian rhythm parameters, a subset of participants underwent actigraph monitoring (Actiwatch 2, Phillips Respironics; Murrysville, PA, USA) that detects body movement with a piezoelectric linear accelerometer and generates a voltage. Subjects wore the Actiwatch 2 on their nondominant wrist for seven consecutive 24-hour periods. Sleep diaries were also completed by these individuals.

\section{Statistical analysis}

Baseline demographic and clinical variables were compared 
across diagnostic groups using analysis of variance for continuous variables and $\chi^{2}$ tests for categorical variables. Neuropsychological variables were compared among diagnostic groups using analysis of covariance, with covariates including age, gender and educational years. All statistical analyses were performed using the IBM SPSS Statistics for Windows, version 23 (IBM Corp., Armonk, NY, USA).

\section{RESULTS}

This section provides an overview of baseline characteristics of the KBASE cohort, and detailed results for specific research interests will be presented in subsequent, more specialized papers.

\section{Study participants}

As of March 2017, in total, 758 individuals had volunteered for the assessment of eligibility for participation in the KBASE cohorts (Figure 1). Among them, 591 individuals were included in the KBASE baseline cohort and 167 individuals were excluded for the following reasons: 1) 63 individuals met the one of the exclusion criteria, such as comorbid medical, psychiatric, or neurological disorder or condition that could affect mental functioning $(n=51)$, presence of severe communication or behavioral problems that would make a clinical examination or brain scan difficult $(n=5)$, contraindications for MRI scans $(\mathrm{n}=6)$, and participation in another clinical trial taking an investigational product $(n=1), 2) 46$ subjects did not meet the inclusion criteria for any diagnostic group, and 3) 58 subjects were excluded due to withdrawal of consent $(n=55)$ or loss of contact $(n=3)$. The 591 individuals in-

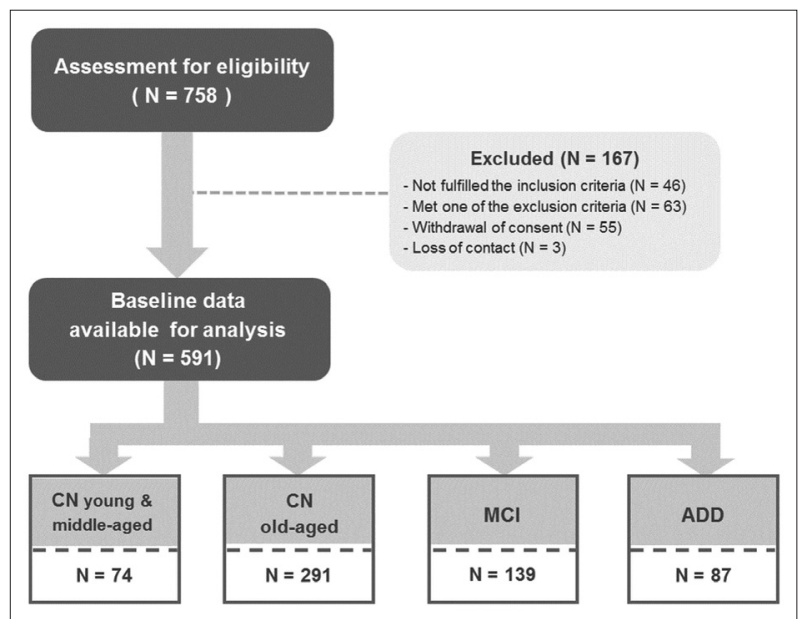

Figure 1. Flow chart describing the composition of the KBASE cohort. CN-ym: Cognitively normal young and middle-aged adults, $\mathrm{CN}$-old: Cognitively normal old-aged adults, $\mathrm{MCl}$ : Mild Cognitive Impairment, ADD: Alzheimer's disease dementia, KBASE: Korean Brain Aging Study for the Early Diagnosis and Prediction of Alzheimer's disease. cluded in the baseline KBASE cohort consisted of $74 \mathrm{CN}-\mathrm{ym}$, 291 CN-old, 139 MCI [amnestic MCI, single domain ( $n=58)$, amnestic MCI, multiple domains $(\mathrm{n}=81)$, and 87 ADD individuals.

\section{Demographic and clinical characteristics}

There were significant group differences in age, gender, and educational levels among the four groups (Table 1). The MCI and ADD individuals were significantly older and more likely to be female compared with the $\mathrm{CN}$-old and $\mathrm{CN}$-ym individuals. Educational years of the $\mathrm{CN}$-old, $\mathrm{MCI}$, and $\mathrm{ADD}$ groups were significantly lower than of the $\mathrm{CN}$-ym group, and those of the MCI and ADD groups were still lower than those of the CN-old group.

Most of the ADD (97\%) and about half of MCI (49\%) participants were referred from special clinics for dementia and cognitive disorder in the two university hospitals in Seoul, South Korea, while $35.3 \%$ of MCI participants were recruited from dementia screening programs at the two public centers for dementia prevention and management. In contrast, the majority of CN-ym and CN-old individuals were recruited from the community through word of mouth or online/offline poster advertisements.

The frequency of FH of ADD in first-degree relatives was the highest in the ADD group (30.2\%), followed by the MCI (23.0\%), CN-old (20.6\%), and CN-ym (8.1\%) groups. The frequency of APOE $\varepsilon 4$ carriers in the ADD group (59.8\%) was also highest among the four groups.

Among 87 individuals with ADD, 33.3\% of them had a global CDR of 0.5 , while the rest had a score of 1 . Compared with the other diagnostic groups, individuals with $\mathrm{ADD}$ showed the highest CDR score in all six domains, whereas the MCI group had intermediate $\mathrm{CDR}$ scores, between $\mathrm{AD}$ and $\mathrm{CN}$ subjects (Table 2). Likewise, BDS-ADL scores and GDS showed similar trends.

When we compared SMCQ and SIRQD scores, the CNold, MCI, and ADD groups scored significantly higher than the $\mathrm{CN}-\mathrm{ym}$, and scores of the MCI and ADD groups were still higher than $\mathrm{CN}$-old. No significant difference in self-reported SMCQ score was found between subjects with MCI and ADD, whereas the SIRQD score was much higher than in the MCI group. Current depressive symptoms, measured by the GDS-KR, CES-D, and HDRS, were significantly higher in subjects with $\mathrm{ADD}$ and $\mathrm{MCI}$ than in $\mathrm{CN}$-ym and $\mathrm{CN}$-old individuals. Additionally, the MCI group showed the highest scores in the GDS-KR and state-anxiety scores, measured by STAI.

\section{Neuropsychological characteristics}

Overall, significant group differences in most neuropsychological measurements among the four diagnostic groups were found after controlling for the effects of age, gender, and edu- 
cational level (Table 3). ADD individuals showed the most severe impairments and MCI individuals showed intermediate scores between ADD and CN-old individuals in most neuropsychological measurements. Compared with the CN-old groups, the MCI and ADD groups showed significant impairments in all tests in verbal and non-verbal memory domains, as well as global cognition (MMSE-KC), semantic and phonemic fluency, confrontational naming test, visuospatial function, executive function, and attention. MCI individuals showed better performance in most neuropsychological tests compared with ADD participants, but impairments in the Word List Immediate Recall, Digit Span Backward, and phonemic fluency tests did not show significant differences compared with ADD participants.

When we compared $\mathrm{CN}$-old and $\mathrm{CN}$-ym groups, $\mathrm{CN}$-old individuals had significantly lower scores in a subset of neuropsychological tests, including the MMSE-KC, immediate and delayed recall of the WMS-IV-K Logical Memory, phone-

Table 1. Baseline demographic characteristics of participants

\begin{tabular}{|c|c|c|c|c|c|}
\hline & $\mathrm{CN}-\mathrm{ym}(\mathrm{N}=74)$ & CN-old $(\mathrm{N}=291)$ & MCI $(\mathrm{N}=139)$ & $\operatorname{ADD}(\mathrm{N}=87)$ & $\mathrm{p}$ \\
\hline Age (years) & $37.7(9.7)$ & $69.2(8.1)$ & $73.7(7.0)$ & $73.0(8.1)$ & $<0.001^{*}$ \\
\hline Age range (years) & $21-54$ & $55-87$ & $57-90$ & $55-87$ & \\
\hline Age frequency & & & & & $<0.001$ \\
\hline$<60$ & $74(100)$ & $49(16.8)$ & $4(2.9)$ & $7(8)$ & \\
\hline $60-69$ & 0 & $97(33.3)$ & $34(24.5)$ & $18(20.7)$ & \\
\hline $70-79$ & 0 & $114(39.2)$ & $71(51.1)$ & $39(44.8)$ & \\
\hline $80-90$ & 0 & $31(10.7)$ & $30(21.6)$ & $23(26.4)$ & \\
\hline Female & $39(52.7)$ & $149(51.2)$ & $92(66.2)$ & $60(69.0)$ & 0.002 \\
\hline Educational years & $15.0(1.9)$ & $11.8(4.8)$ & $10.0(4.5)$ & $9.1(5.4)$ & $<0.001^{*}$ \\
\hline Education level & & & & & $<0.001$ \\
\hline$\leq 12$ years & $12(16.2)$ & $172(59.1)$ & $111(79.9)$ & $69(79.3)$ & \\
\hline$>12$ years & $62(83.8)$ & $119(40.9)$ & $28(20.1)$ & $18(20.7)$ & \\
\hline Marital status & & & & & $<0.001$ \\
\hline Married & $45(60.8)$ & $231(79.4)$ & $116(83.5)$ & $64(73.6)$ & \\
\hline Widowed & $1(1.4)$ & $44(15.1)$ & $20(14.4)$ & $19(21.8)$ & \\
\hline Separation or divorced & $2(2.7)$ & $13(4.5)$ & $2(1.4)$ & $3(3.4)$ & \\
\hline Unmarried & $26(35.1)$ & $2(0.7)$ & 0 & 0 & \\
\hline Others & 0 & $1(0.3)$ & $1(0.7)$ & $1(1.1)$ & \\
\hline Recruitment & & & & & $<0.001$ \\
\hline Referral from dementia clinic & 0 & $5(1.7)$ & $68(48.9)$ & $84(96.6)$ & \\
\hline Referral from local dementia screening program & 0 & $10(3.4)$ & $49(35.3)$ & $1(1.1)$ & \\
\hline Online and offline poster & $18(24.3)$ & $17(5.8)$ & $3(2.2)$ & 0 & \\
\hline Word of mouth ${ }^{\dagger}$ & $56(75.7)$ & $259(89.0)$ & $19(13.7)$ & $2(2.3)$ & \\
\hline $\mathrm{FH}$ of $\mathrm{AD}$ dementia in 1st degree relatives & $6(8.1)$ & $60(20.6)$ & $30(21.9)$ & $24(28.2)$ & 0.016 \\
\hline APOE genotype $e^{\ddagger}$ & & & & & $<0.001$ \\
\hline$\varepsilon 2 / \varepsilon 2$ & $0(0)$ & $2(0.7)$ & $0(0)$ & $0(0)$ & \\
\hline$\varepsilon 2 / \varepsilon 3$ & $9(12.2)$ & $28(9.7)$ & $10(7.2)$ & $2(2.3)$ & \\
\hline$\varepsilon 3 / \varepsilon 3$ & $44(59.5)$ & $207(71.4)$ & $82(59.0)$ & $33(37.9)$ & \\
\hline$\varepsilon 2 / \varepsilon 4$ & $2(2.7)$ & $2(0.7)$ & $3(2.2)$ & $0(0)$ & \\
\hline$\varepsilon 3 / \varepsilon 4$ & $17(23.0)$ & 49 (16.9) & $34(24.5)$ & $40(46.0)$ & \\
\hline$\varepsilon 4 / \varepsilon 4$ & $2(2.7)$ & $2(0.7)$ & $10(7.2)$ & $12(13.8)$ & \\
\hline APOE e4 carrier status $\ddagger$ & $21(28.4)$ & $53(18.3)$ & $47(33.8)$ & $52(59.8)$ & $<0.001$ \\
\hline
\end{tabular}

Data are shown as mean (SD) or N (\%). ${ }^{*} \mathrm{CN}-\mathrm{ym}<\mathrm{CN}$-old $<\mathrm{MCI}$, ADD in post-hoc analysis, ${ }^{\dagger}$ recommended by other participants, family members, friends, or acquaintances, $\ddagger_{\text {data }}$ for $1 \mathrm{CN}$-old subjects was unavailable. $\mathrm{CN}$-ym: Cognitively normal young and middle-aged adults, CN-old: Cognitively normal old-aged adults, MCI: Mild Cognitive Impairment, ADD: Alzheimer's disease dementia, FH: Family history, APOE: Apolipotrotein E 
mic fluency, and the WAIS-IV-K Block Design.

\section{Medical comorbidities and anthropometric measurements}

Compared with the $\mathrm{CN}$-ym group, the $\mathrm{CN}$-old, $\mathrm{MCI}$, and $\mathrm{ADD}$ groups were more likely to have hypertension, diabetes mellitus, and hyperlipidemia, and to have higher VRS (Table 4). However, no significant group difference in the frequency of each VRF or VRS was observed among CN-old, MCI, and $\mathrm{ADD}$ groups. Compared with the $\mathrm{CN}$-ym group, both $\mathrm{SBP}$ and DBP were higher in the $\mathrm{CN}$-old, $\mathrm{MCI}$, and ADD groups. No significant group difference was found in BMI or waist circumferences among the four groups. Detailed findings on medical comorbidities, ABI, and other anthropometric measurements will be described in more specialized papers.

\section{Neuroimaging}

As of March 2017, baseline $\left[{ }^{11} \mathrm{C}\right] \mathrm{PiB}-\mathrm{PET},\left[{ }^{18} \mathrm{~F}\right] \mathrm{FDG}-\mathrm{PET}$, and MRI/MRA images from 574 individuals were available for analyses. Three subjects were waiting for scheduled scans and 14 subjects were excluded from further image analyses for the following reasons: 1) did not participate in $\left[{ }^{11} \mathrm{C}\right] \mathrm{PiB}-$ $\mathrm{PET} / \mathrm{MR}$ scan due to general medical condition, withdrawal of consent, or lack of cooperation $(n=12)$ and 2$)$ unable to use images due to severe artifacts $(n=2)$. Detailed results of neuroimaging data will be presented in more specialized papers.

\section{Actigraph monitoring}

In total, 275 participants underwent actigraph monitoring for seven consecutive 24-h periods. Among them, 40 were $\mathrm{CN}$-ym, 153 were CN-old, 46 were $\mathrm{MCI}$, and 36 were ADD participants. Detailed results of analyses from actigraph monitoring data will be presented in more specialized papers.

\section{DISCUSSION}

The KBASE is an ongoing prospective, longitudinal cohort study that recruited individuals with a wide range of ages (20-90 years) and cognitive status (CN, MCI, and ADD) to discover novel biomarkers for the early detection of in vivo $\mathrm{AD}$ pathology and to reveal the influence of various lifetime experiences and bodily changes on $\mathrm{AD}$-related brain chang-

Table 2. Baseline clinical characteristics of participants

\begin{tabular}{|c|c|c|c|c|c|}
\hline & $\mathrm{CN}-\mathrm{ym}$ & CN-old & MCI & $\mathrm{ADD}$ & $\mathrm{p}$ \\
\hline \multicolumn{6}{|l|}{ CDR domain } \\
\hline Memory & $0(0)$ & $0(0)$ & $0.50(0.04)$ & $1.14(0.37)$ & $<0.001^{*}$ \\
\hline Orientation & $0(0)$ & $0.00(0.04)$ & $0.43(0.19)$ & $1.03(0.44)$ & $<0.001^{*}$ \\
\hline Judgment & $0(0)$ & $0.00(0.04)$ & $0.21(0.25)$ & $0.86(0.29)$ & $<0.001^{*}$ \\
\hline Community affairs & $0(0)$ & $0(0)$ & $0.19(0.24)$ & $0.94(0.25)$ & $<0.001^{*}$ \\
\hline Home and hobbies & $0(0)$ & $0(0)$ & $0.17(0.24)$ & $0.77(0.31)$ & $<0.001^{*}$ \\
\hline Personal care & $0(0)$ & $0(0)$ & $0.00(0.04)$ & $0.31(0.48)$ & $<0.001^{*}$ \\
\hline Global CDR & & & & & $<0.001$ \\
\hline CDR 0 & $74(100)$ & $291(100)$ & 0 & 0 & \\
\hline CDR 0.5 & 0 & 0 & $139(100)$ & $29(33.3)$ & \\
\hline CDR 1 & 0 & 0 & 0 & $58(66.7)$ & \\
\hline CDR-SB & $0(0)$ & $0.01(0.06)$ & $1.50(0.65)$ & $5.05(1.45)$ & $<0.001^{*}$ \\
\hline GDS & $1.00(0.00)$ & $1.05(0.21)$ & $2.93(0.35)$ & $4.53(0.50)$ & $<0.001^{\dagger}$ \\
\hline BDS-ADL & $0(0)$ & $0.01(0.06)$ & $1.28(0.61)$ & $3.83(1.09)$ & $<0.001^{\dagger}$ \\
\hline SMCQ & $0.99(1.27)$ & $2.14(1.95)$ & $5.60(3.11)$ & $5.66(3.05)$ & $<0.001^{*}$ \\
\hline SIRQD & $1.45(2.51)$ & $3.54(3.89)$ & $9.54(6.26)$ & $16.99(6.24)$ & $<0.001^{\dagger}$ \\
\hline GDS-KR & $4.43(3.91)$ & $4.73(4.94)$ & $10.27(6.98)$ & $7.93(5.50)$ & $<0.001^{\ddagger}$ \\
\hline HRSD & $0.7(1.8)$ & $0.8(1.6)$ & $3.1(4.2)$ & $2.0(2.8)$ & $<0.001^{\S}$ \\
\hline CES-D $\pi$ & $26.0(6.2)$ & $28.2(6.8)$ & $33.7(9.9)$ & $32.3(9.9)$ & $<0.001^{\S}$ \\
\hline STAI-stateף & $34.8(8.6)$ & $35.4(9.9)$ & $40.5(11.8)$ & $38.4(11.5)$ & $<0.001^{\| \prime}$ \\
\hline
\end{tabular}

Data are shown as mean (SD) or N (\%). Significant findings from post-hoc analyses of each variable are listed below. ${ }^{*} \mathrm{CN}-\mathrm{ym}, \mathrm{CN}-\mathrm{old}<\mathrm{MCI}<$ $\mathrm{ADD},{ }^{\dagger} \mathrm{CN}$-ym<CN-old<MCI<ADD, ${ }^{\ddagger} \mathrm{CN}$-ym, CN-old $<\mathrm{ADD}<\mathrm{MCI},{ }^{\circledR} \mathrm{CN}$-ym, CN-old<MCI, ADD, "CN-ym, CN-old<MCI, "data for 7 subjects (3 CN old, $1 \mathrm{MCI}, 3 \mathrm{ADD}$ ) were unavailable. CN-ym: Cognitively normal young and middle-aged adults, CN-old: Cognitively normal old-aged adults, MCI: Mild Cognitive Impairment, ADD: Alzheimer's disease dementia, CDR: Clinical dementia rating, CDR-SB: CDR sum of box, GDS: Global deterioration scale, BDS-ADL: Blessed Dementia Scale-Activity of Daily Living, SMCQ: Subjective Memory Complaints Questionnaire, SIRQD: Seoul Informant Report Questionnaire for Dementia, STAI: State-Trait Anxiety Inventory 
es, such as A $\beta$ deposition and neuronal injury. As of March 2017, almost 600 individuals underwent the baseline multidisciplinary assessment including comprehensive clinical and neuropsychological assessments, multi-modal imaging including $\left[{ }^{11} \mathrm{C}\right] \mathrm{PiB}-\mathrm{PET},\left[{ }^{18} \mathrm{~F}\right] \mathrm{FDG}-\mathrm{PET}$, MRI and MRA, and blood laboratory tests according to a standardized protocol.

Table 3. Baseline neuropsychological characteristics of participants

\begin{tabular}{|c|c|c|c|c|c|}
\hline & $\mathrm{CN}-\mathrm{ym}$ & CN-old & MCI & $\mathrm{ADD}$ & $\mathrm{p}$ \\
\hline MMSE-KC & $29.08(0.99)$ & $26.90(2.61)$ & $22.48(3.16)$ & $16.39(4.17)$ & $<0.001^{*}$ \\
\hline Semantic fluency & $19.65(4.31)$ & $15.69(4.77)$ & $11.97(3.80)$ & $8.77(3.73)$ & $<0.001^{\dagger}$ \\
\hline Boston naming test & $13.41(0.99)$ & $12.14(2.19)$ & $9.96(2.48)$ & $8.53(3.15)$ & $<0.001^{\ddagger}$ \\
\hline \multicolumn{6}{|l|}{ Verbal memory } \\
\hline Word list: immediate recall & $24.12(3.54)$ & $19.45(4.16)$ & $9.20(6.36)$ & $9.64(4.42)$ & $<0.001 \S$ \\
\hline Word list: delayed recall & $8.77(1.43)$ & $6.53(1.85)$ & $2.75(1.78)$ & $0.86(1.44)$ & $<0.001^{\dagger}$ \\
\hline Word list: recognition & $9.84(0.64)$ & $9.11(1.22)$ & $6.30(2.66)$ & $3.91(2.71)$ & $<0.001^{\dagger}$ \\
\hline Constructional praxis & $10.85(0.49)$ & $10.07(1.33)$ & $9.20(1.52)$ & $8.33(2.32)$ & $<0.001^{\dagger}$ \\
\hline Nonverbal memory delayed recall & $10.23(1.53)$ & $7.41(2.86)$ & $2.49(2.59)$ & $0.76(1.32)$ & $<0.001^{\dagger}$ \\
\hline \multicolumn{6}{|l|}{ Stroop Test ${ }^{1 \| \prime}$} \\
\hline Word reading & $95.23(11.46)$ & $76.96(18.41)$ & $62.85(17.86)$ & $53.71(19.13)$ & $<0.001^{\|}$ \\
\hline Color reading & $79.10(11.45)$ & $62.54(13.27)$ & $52.01(13.06)$ & $42.56(15.20)$ & $<0.001^{\dagger}$ \\
\hline Color-word reading & $55.35(11.46)$ & $37.70(11.10)$ & $28.25(10.90)$ & $21.08(12.38)$ & $<0.001^{\dagger}$ \\
\hline \multicolumn{6}{|l|}{ WMS-IV-K logical memory ${ }^{\|1\|}$} \\
\hline Immediate recall & $29.73(7.79)$ & $19.12(7.34)$ & $9.24(5.68)$ & $3.76(5.02)$ & $<0.001^{*}$ \\
\hline Delayed recall & $26.57(7.93)$ & $13.87(7.35)$ & $3.87(4.56)$ & $0.68(4.25)$ & $<0.001^{*}$ \\
\hline Recognition & $25.65(3.06)$ & $21.11(3.96)$ & $16.74(3.00)$ & $14.96(2.82)$ & $<0.001^{\dagger}$ \\
\hline \multicolumn{6}{|l|}{$\mathrm{RCFT}^{\|\|}$} \\
\hline Copy & $34.03(2.45)$ & $29.59(6.19)$ & $24.68(8.37)$ & $17.80(11.48)$ & $<0.001^{\|, \pi}$ \\
\hline 3-minute delay & $21.80(5.57)$ & $14.10(6.44)$ & $6.61(5.91)$ & $1.46(3.53)$ & $<0.001^{\dagger}$ \\
\hline 30-minute delay & $21.87(5.84)$ & $13.63(6.52)$ & $5.64(5.60)$ & $0.75(3.43)$ & $<0.001^{\dagger}$ \\
\hline \multicolumn{6}{|l|}{ Digit Span } \\
\hline Forward & $9.77(2.58)$ & $6.97(2.58)$ & $5.59(2.29)$ & $5.55(2.53)$ & 0.054 \\
\hline Backward & $7.93(2.73)$ & $4.96(2.08)$ & $3.77(1.53)$ & $3.25(2.02)$ & $<0.001 \S$ \\
\hline COWAT (phonemic fluency) total"II & $40.01(12.27)$ & $25.60(11.44)$ & $19.28(9.04)$ & $16.25(10.01)$ & $<0.001^{* *}$ \\
\hline WAIS-IV-K block design"III & $48.45(10.03)$ & $29.99(9.43)$ & $23.78(8.17)$ & $17.57(10.01)$ & $<0.001^{*}$ \\
\hline \multicolumn{6}{|l|}{ Clock Drawing Test ${ }^{\|\prime\|}$} \\
\hline CLOX 1 & $13.27(1.55)$ & $13.15(2.10)$ & $11.86(3.31)$ & $9.01(4.43)$ & $<0.001^{11,+\dagger}$ \\
\hline CLOX 2 & $14.72(0.69)$ & $14.50(0.93)$ & $13.71(1.92)$ & $11.84(3.47)$ & $<0.001^{11, \text { †† }}$ \\
\hline \multicolumn{6}{|l|}{ Trail Making Test ${ }^{\| \prime \prime}$} \\
\hline Trails A (time, sec) & $28.65(20.83)$ & $49.89(23.29)$ & $76.93(44.70)$ & $101.91(65.84)$ & $<0.001^{\ddagger \ddagger}$ \\
\hline Trails B (time, sec) & $63.66(26.30)$ & $124.18(58.46)$ & $184.88(64.40)$ & $176.08(72.25)$ & $<0.001 \S \S$ \\
\hline KART ${ }^{\|\|}$ & $47.87(2.48)$ & $44.89(5.03)$ & $42.39(6.97)$ & $39.67(9.37)$ & $<0.001^{\|}$ \\
\hline
\end{tabular}

Data are shown as mean (SD). Analysis of covariance (ANCOVA) was performed with age, sex, and educational years as covariates. Significant findings from post-hoc analyses are listed below. ${ }^{*} \mathrm{CN}$-ym $>\mathrm{CN}$-old $>\mathrm{MCI}>\mathrm{ADD},{ }^{\dagger} \mathrm{CN}$-ym, CN-old $>\mathrm{MCI}>\mathrm{ADD},{ }^{\ddagger} \mathrm{CN}$-old $>\mathrm{CN}$-ym, $\mathrm{MCI}>\mathrm{ADD},{ }^{\S} \mathrm{CN}$-ym, $\mathrm{CN}$-old $>\mathrm{MCI}, \mathrm{ADD}, " \mathrm{CN}$-old $>\mathrm{MCI}>\mathrm{ADD},{ }^{\top} \mathrm{CN}$-ym, $\mathrm{CN}$-old $>\mathrm{ADD},{ }^{* *} \mathrm{CN}$-ym $>\mathrm{CN}$-old $>\mathrm{MCI}, \mathrm{ADD},{ }^{+\dagger} \mathrm{CN}-\mathrm{ym}>\mathrm{ADD}$

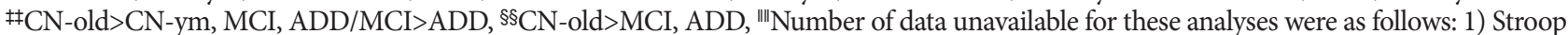
Test: Word Reading (2 ADD), Color Reading (3 ADD), Color-Word Reading (4 ADD), 2) WMS-IV-K Logical Memory: Immediate Memory (2 MCI, 1 ADD), Delayed recall (2 MCI, 2 ADD), Recognition (1 CN-old, 6 MCI, 15 ADD), 3) RCFT (1 MCI, 3 ADD), 4) Digit Span: Forward (1 MCI, $1 \mathrm{ADD})$, Backward (1 MCI, 3 ADD), 5) COWAT (1 MCI, 11 ADD), 6) WAIS-IV-K Block Design: (1 CN-ym, 1 MCI, 6 ADD), 7) Clock Drawing Test; CLOX1 (1 CN-old, 1 MCI, 2 ADD), CLOX 2 (1 CN old, 1 ADD), 8) Trail Making Test; Trails A (3 CN-old, 4 MCI, 18 ADD), Trails B (3 CN-ym, $111 \mathrm{CN}$-old, $105 \mathrm{MCI}, 75 \mathrm{ADD})$, and 9) KART (11 CN-ym, $11 \mathrm{CN}$-old, 19 MCI, 21 ADD). CN-ym: Cognitively normal young and middle-aged adults, CN-old: Cognitively normal old-aged adults, MCI: Mild Cognitive Impairment, ADD: Alzheimer's disease dementia, MMSE-KC: Mini-Mental State Examination in the Korean version of Consortium to Establish a Registry for Alzheimer's Disease, WMS-IV-K: Wechsler Memory Scale-Fourth edition Korean version, RCFT: Rey Complex Figure Test, WAIS-IV-K: Wechsler Adult Intelligence Scale-Fourth edition Korean version, KART: Korean Adult Reading Test 
Table 4. Medical comorbidities, blood pressure, and anthropometric measurements

\begin{tabular}{|c|c|c|c|c|c|}
\hline & $\mathrm{CN}-\mathrm{ym}$ & CN-old & MCI & $\mathrm{ADD}$ & $\mathrm{p}$ \\
\hline Hypertension & $4(5.4)$ & $136(46.7)$ & $74(53.2)$ & $39(44.8)$ & $<0.001$ \\
\hline Diabetes mellitus & $3(4.1)$ & $48(16.5)$ & $26(18.7)$ & $14(16.1)$ & 0.027 \\
\hline Coronary heart disease & $0(0)$ & $16(5.5)$ & $5(3.6)$ & $5(5.7)$ & 0.098 \\
\hline Hyperlipidemia & $1(1.4)$ & $99(34.0)$ & $51(36.7)$ & $29(33.3)$ & $<0.001$ \\
\hline Stroke & $0(0)$ & $0(0)$ & $0(0)$ & $0(0)$ & - \\
\hline TIA & $0(0)$ & $2(0.7)$ & $1(0.7)$ & $0(0)$ & 0.770 \\
\hline VRS & $1.8(5.9)$ & $17.2(16.1)$ & $18.8(16.8)$ & $16.7(16.5)$ & $<0.001$ \\
\hline SBP-supine, mm Hg* & $114.5(16.7)$ & $124.6(16.5)$ & $128.1(17.5)$ & $124.9(17.7)$ & $<0.001$ \\
\hline DBP-supine, mm Hg* & $71.8(12.7)$ & $76.5(11.1)$ & $76.4(10.5)$ & $76.3(12.5)$ & 0.015 \\
\hline Height, $\mathrm{cm}$ & $166.6(8.1)$ & $160.1(8.8)$ & $155.9(8.1)$ & $155.6(8.7)$ & $<0.001$ \\
\hline Weight, kg & $67.1(14.0)$ & $62.2(9.9)$ & $60.0(9.2)$ & $58.1(10.0)$ & $<0.001$ \\
\hline BMI, $\mathrm{kg} / \mathrm{m}^{2}$ & $24.0(3.9)$ & $24.2(3.0)$ & $24.7(3.1)$ & $24.0(3.7)$ & 0.378 \\
\hline Waist circumference, $\mathrm{cm}^{*}$ & $84.5(9.1)$ & $86.5(7.5)$ & $87.1(8.0)$ & $85.5(7.6)$ & 0.105 \\
\hline
\end{tabular}

Data are shown as N (\%). *data for $1 \mathrm{CN}$-old subject was unavailable. $\mathrm{CN}$-ym: Cognitively normal young and middle-aged adults, $\mathrm{CN}$-old: Cognitively normal old-aged adults, MCI: Mild Cognitive Impairment, ADD: Alzheimer's disease dementia, TIA: transient ischemic attack, VRS: vascular risk factor score, SBP: systolic blood pressure, DBP: diastolic blood pressure, BMI: body mass index

Additionally, more than 270 participants underwent objective sleep-circadian rhythm measurements using actigraph monitoring at baseline. To the best of our knowledge, this is the largest prospective cohort study to measure both $\mathrm{A} \beta$ and neurodegeneration biomarkers, and to comprehensively evaluate various lifetime experiences and bodily status-related variables in wellcharacterized $\mathrm{CN}, \mathrm{MCI}$, and ADD individuals in an Asian (Korean) population.

Although KBASE recruited ADD participants with very mild to mild stage (CDR 0.5 and 1), the ADD group showed the most severe cognitive and functional impairments among all groups, consistent with previous reports. ${ }^{60,61}$ Additionally, we could confirm the previously known demographic risk factors of $\mathrm{AD}$ in this cohort such as older age, gender (i.e., female), low education, and family history of AD and the APOE e4 allele. ${ }^{1,3}$ About $60 \%$ of ADD group had at least one or more APOE e4 allele, which was the highest among all groups and consistent with previous reports. ${ }^{62}$

For the MCI group in the KBASE cohort, only amnestic MCI subjects who met the core clinical criteria for MCI, recommended by NIA-AA, ${ }^{28}$ were included to maximize the proportion of MCI with positive $A \beta$ biomarkers, given the lower probability of having $\mathrm{AD}$ pathology in non-amnestic MCI versus amnestic MCI. ${ }^{63}$ As expected, most of baseline CDR scores and neuropsychological characteristics of MCI individuals had intermediate values between $\mathrm{CN}$ and $\mathrm{ADD}$, consistent with previous studies. ${ }^{60,61}$ Additionally, individuals in the MCI group were significantly older than the $\mathrm{CN}$-old group, more likely to be female, and their educational level was lower than the $\mathrm{CN}$ groups, indicating that the $\mathrm{MCI}$ group in the KBASE cohort well captured previously reported risk factors for $\mathrm{AD} .{ }^{1,3}$

The KBASE cohort purposefully recruited a large number of $\mathrm{CN}$ subjects to obtain normative information on various measurements to improve the clinical utility of the biomarkers and the interpretation of results at the individual level. Thus, the proportion of $\mathrm{CN}$ subjects was the largest in the KBASE cohort. Additionally, we included young and middle-aged $\mathrm{CN}$ as well as old-aged $\mathrm{CN}$ subjects in order to distinguish the influences of normal and pathological brain aging. When we compared the two $\mathrm{CN}$ groups, the educational level of the $\mathrm{CN}$ old individuals was lower than that of the $\mathrm{CN}$-ym subjects, with a large variance. This result is consistent with a previous report of elderly Korean populations, ${ }^{29}$ which might be related to a birth cohort effect because the educational attainment of the Korean people generally has improved dramatically over the past several decades. ${ }^{64,65}$ Thus, the differences in educational level should be considered in the accurate interpretation of neuropsychological tests. Additionally, the data from the KBASE cohort can be used to investigate whether discrepancies in educational levels, sociocultural environments, or race/ethnicity differentially contribute to $\mathrm{AD}$ processes and to validate the findings from similar large-scale, neuroimaging based-cohorts such as the Alzheimer's Disease Neuroimaging Initiative $(\mathrm{ADNI})^{60}$ and the Australian Imaging, Biomarkers \& Lifestyle Flagship Study of Ageing (AIBL), ${ }^{61}$ which were mostly composed of highly educated Caucasians.

This study has several strengths. First, in contrast to multicenter studies like ADNI, all baseline neuroimaging and blood laboratory tests were conducted at a single center in a university hospital in South Korea (SNUH), which contributes sig- 
nificantly to reducing inter-center variability as well as cost and effort in study management. Second, a simultaneous PETMR machine was used for the acquisition of various kinds of multi-modal imaging, which is another methodological strength that contributes to the accuracy of image co-registration in technical aspects and to improved convenience for the participants by reducing scanning time and reducing exposure to radiation.

Furthermore, one of characteristics of the KBASE cohort that is distinct from other large-size neuroimaging based cohorts (e.g., ADNI or AIBL) is that we recruited $\mathrm{CN}$ individuals with a wide age range, young adulthood to late life (from 20 to 90 years old). A cross-sectional analysis between CNym and $\mathrm{CN}$-old groups, and longitudinal follow-up of $\mathrm{CN}$ individuals will enable us to investigate the nature of both normal and pathological brain aging at the beginning and to discover novel protective or risk factors in certain windows of life that contribute to the development of $\mathrm{AD}$ pathologies. Additionally, because we did not selectively include participants with the APOE $\varepsilon 4$ carrier or positive $\mathrm{FH}$ of $\mathrm{AD}$, generalizability of the findings from this cohort will be less compromised than the other cohort studies that selectively recruited at-risk populations of $\mathrm{AD}$ for amyloid imaging. ${ }^{63,66}$

There are some potential limitations. First, the number of participants in certain age groups (e.g., 80-90 years) is still limited, which might reduce statistical power when comparing the oldest-old CN individuals with other groups or conducting an analysis within the age group. Second, although most of the $\mathrm{CN}$ volunteers were recruited from a community setting, these volunteers were not random and might have been more motivated and interested in their health and, therefore, likely to be physically healthier than the general population.

At present, cross-sectional analyses of the baseline data are ongoing and will be presented with more details in specialized papers. Briefly, the association of $\mathrm{A} \beta$ and neuronal injury biomarkers of $\mathrm{AD}$ with numerous variables from the comprehensive clinical and neuropsychological assessments, personality and lifetime experiences, and blood laboratory tests are under analysis and being prepared for manuscripts. Proteomic, biochemical and genetic analyses using blood and DNA specimen are also ongoing. Additionally, external validity of candidate biomarkers will be tested by using the data and samples collected from an independent cohort that is established for validation of candidate biomarkers discovered in the KBASE cohort.

Currently, participants in the KBASE cohort are followed annually with clinical and neuropsychological assessments, and biannually with full KBASE assessments, including neuroimaging and blood laboratory tests (Supplementary Table 1, Supplementary Materials 1 in the online-only Data Sup- plement). Furthermore, among participants who completed baseline assessments, tau PET using ${ }^{18} \mathrm{~F}-\mathrm{AV}$-1451 tracers will be added to the biannual assessment protocol for 210 individuals. Additional multidisciplinary research to investigate the associations of in vivo AD pathologies with retinal changes, hearing difficulties, metabolic signatures in hair samples, and sleep parameters of polysomnography are also ongoing collaboratively between intra- and extramural research teams. Further analyses of longitudinal follow-up data from the KBASE will contribute to the development of predictive biomarkers related to AD-specific brain changes in the future and to the identification of causal relationships between lifetime experiences or bodily changes and the development of AD pathologies.

\section{Supplementary Materials}

The online-only Data Supplement is available with this article at https://doi.org/10.4306/pi.2017.14.6.851.

\section{Acknowledgments}

This study was supported by a grant from Ministry of Science, ICT and Future Planning (Grant No: NRF-2014M3C7A1046042). The funding source had no role in the study design, data collection, data analysis, data interpretation, writing of the manuscript or decision to submit it for publication.

\section{REFERENCES}

1. Prince M, Albanese E, Guerchet M, Prina M. World Alzheimer Report 2014: Dementia and Risk Reduction-An Analysis of Protective and Modifiable Factors London: Alzheimer's Disease International (ADI); 2014.

2. Winblad B, Amouyel P, Andrieu S, Ballard C, Brayne C, Brodaty H, et al. Defeating Alzheimer's disease and other dementias: a priority for European science and society. Lancet Neurol 2016;15:455-532.

3. Qiu C, Kivipelto M, von Strauss E. Epidemiology of Alzheimer's disease: occurrence, determinants, and strategies toward intervention. Dialogues Clin Neurosci 2009;11:111-128.

4. Brookmeyer R, Johnson E, Ziegler-Graham K, Arrighi HM. Forecasting the global burden of Alzheimer's disease. Alzheimers Dement 2007; 3:186-191.

5. Brookmeyer R, Gray S, Kawas C. Projections of Alzheimer's disease in the United States and the public health impact of delaying disease onset. Am J Public Health 1998;88:1337-1342.

6. Zissimopoulos J, Crimmins E, St Clair P. The value of delaying Alzheimer's disease onset. Forum Health Econ Policy 2014;18:25-39.

7. Doody RS, Thomas RG, Farlow M, Iwatsubo T, Vellas B, Joffe S, et al. Phase 3 trials of solanezumab for mild-to-moderate Alzheimer's disease. N Engl J Med 2014;370:311-321.

8. Hardy J, De Strooper B. Alzheimer's disease: where next for anti-amyloid therapies? Brain 2017; 140:853-855.

9. Graham WV, Bonito-Oliva A, Sakmar TP. Update on Alzheimer's disease therapy and prevention strategies. Annu Rev Med 2017;68:413-430.

10. Sperling RA, Jack CR Jr, Aisen PS. Testing the right target and right drug at the right stage. Sci Transl Med 2011;3:111-133.

11. Epelbaum S, Genthon R, Cavedo E, Habert MO, Lamari F, Gagliardi G, et al. Preclinical Alzheimer's disease: a systematic review of the cohorts underlying the concept. Alzheimers Dement 2017;13:454-467.

12. Karlawish J, Jack CR Jr, Rocca WA, Snyder HM, Carrillo MC. Alzheimer's disease: the next frontier-Special Report 2017. Alzheimers Dement 
2017;13:374-380.

13. Humpel C. Identifying and validating biomarkers for Alzheimer's disease. Trends Biotechnol 2011;29:26-32.

14. Jack CR Jr, Albert MS, Knopman DS, McKhann GM, Sperling RA, Carrillo MC, et al. Introduction to the recommendations from the $\mathrm{Na}$ tional Institute on Aging-Alzheimer's Association workgroups on diagnostic guidelines for Alzheimer's disease. Alzheimers Dement 2011; 7:257-262.

15. McKhann GM, Knopman DS, Chertkow H, Hyman BT, Jack CR Jr, Kawas $\mathrm{CH}$, et al. The diagnosis of dementia due to Alzheimer's disease: recommendations from the National Institute on Aging-Alzheimer's Association workgroups on diagnostic guidelines for Alzheimer's disease. Alzheimers Dement 2011;7:263-269.

16. Dubois B, Feldman HH, Jacova C, Hampel H, Molinuevo JL, Blennow $\mathrm{K}$, et al. Advancing research diagnostic criteria for Alzheimer's disease: the IWG-2 criteria. Lancet Neurol 2014;13:614-629.

17. Henriksen K, O’Bryant SE, Hampel H, Trojanowski JQ, Montine TJ, Jeromin A, et al. The future of blood-based biomarkers for Alzheimer's disease. Alzheimers Dement 2014;10:115-131.

18. Fourier A, Portelius E, Zetterberg H, Blennow K, Quadrio I, Perret-Liaudet A. Pre-analytical and analytical factors influencing Alzheimer's disease cerebrospinal fluid biomarker variability. Clin Chim Acta 2015; 449:9-15.

19. Johnson KA, Fox NC, Sperling RA, Klunk WE. Brain imaging in Alzheimer disease. Cold Spring Harb Perspect Med 2012;2:a006213.

20. Blennow K. Biomarkers in Alzheimer's disease drug development. Nat Med 2010;16:1218-1222.

21. McEvoy LK, Brewer JB. Quantitative structural MRI for early detection of Alzheimer's disease. Expert Rev Neurother 2010;10:1675-1688.

22. Thambisetty M, Lovestone S. Blood-based biomarkers of Alzheimer's disease: challenging but feasible. Biomark Med 2010;4:65-79.

23. O’Bryant SE, Mielke MM, Rissman RA, Lista S, Vanderstichele H, Zetterberg $\mathrm{H}$, et al. Blood-based biomarkers in Alzheimer disease: current state of the science and a novel collaborative paradigm for advancing from discovery to clinic. Alzheimers Dement 2017;13:45-58.

24. Karch CM, Cruchaga C, Goate AM. Alzheimer's disease genetics: from the bench to the clinic. Neuron 2014;83:11-26.

25. Bertram L. Next Generation Sequencing in Alzheimer's Disease. In: Castrillo JI, Oliver SG, Editors. Systems Biology of Alzheimer's Disease. New York, NY: Springer New York, 2016, p.281-297.

26. Wang HX, MacDonald SW, Dekhtyar S, Fratiglioni L. Association of lifelong exposure to cognitive reserve-enhancing factors with dementia risk: a community-based cohort study. PLoS Med 2017;14:e1002251.

27. Stern Y. Cognitive reserve in ageing and Alzheimer's disease. Lancet Neurol 2012;11:1006-1012.

28. Albert MS, DeKosky ST, Dickson D, Dubois B, Feldman HH, Fox NC, et al. The diagnosis of mild cognitive impairment due to Alzheimer's disease: recommendations from the National Institute on Aging-Alzheimer's Association workgroups on diagnostic guidelines for Alzheimer's disease. Alzheimers Dement 2011;7:270-279.

29. Lee DY, Lee KU, Lee JH, Kim KW, Jhoo JH, Kim SY, et al. A normative study of the CERAD neuropsychological assessment battery in the Korean elderly. J Int Neuropsychol Soc 2004;10:72-81.

30. American Psychiatric Association. Diagnostic and Statistical Manual of Mental Disorderss: DSM-IV-TR. Washington, DC: American Psychiatric Association; 2000.

31. Morris JC, Heyman A, Mohs RC, Hughes JP, van Belle G, Fillenbaum G, et al. The Consortium to Establish a Registry for Alzheimer's Disease (CERAD). Part I. Clinical and neuropsychological assessment of Alzheimer's disease. Neurology 1989;39:1159-1165.

32. Lee JH, Lee KU, Lee DY, Kim KW, Jhoo JH, Kim JH, et al. Development of the Korean version of the Consortium to Establish a Registry for Alzheimer's Disease Assessment Packet (CERAD-K): clinical and neuropsychological assessment batteries. J Gerontol B Psychol Sci Soc Sci 2002;57:P47-P53.
33. Morris JC. The Clinical Dementia Rating (CDR): current version and scoring rules. Neurology 1993;43:2412-2414.

34. Blessed G, Tomlinson BE, Roth M. The association between quantitative measures of dementia and of senile change in the cerebral grey matter of elderly subjects. Br J Psychiatry 1968;114:797-811.

35. First MB, Spitzer RL, Gibbon M, Williams JBW. Structured Clinical Interview for DSM-IV-TR Axis I Disorders, Research Version, Patient Edition. (SCID-I/P). New York: Biometrics Research, New York State Psychiatric Institute; 2002.

36. Mulsant BH, Sweet R, Rifai AH, Pasternak RE, McEachran A, Zubenko GS. The use of the Hamilton Rating Scale for Depression in elderly patients with cognitive impairment and physical illness. Am J Geriatr Psychiatry 1994;2:220-229.

37. Yesavage JA, Brink TL, Rose TL, Lum O, Huang V, Adey M, et al. Development and validation of a geriatric depression screening scale: a preliminary report. J Psychiatr Res 1982-1983;17:37-49.

38. Bae JN, Cho MJ. Development of the Korean version of the Geriatric Depression Scale and its short form among elderly psychiatric patients. J Psychosom Res 2004;57:297-305.

39. Cho MJ, Kim KH. Diagnostic validity of the CES-D (Korean Version) in the Assessment of DSM-III-R Major Depression. J Korean Neuropsychiatr Assoc 1993;32:381-399.

40. Spielberger CD, Gorsuch RL, Lushene R, Vagg PR, Jacobs GA. Manual for the State-Trait Anxiety Inventory (STAI). Palo Alto, CA: Consulting Psychologists Press; 1983.

41. Youn JC, Kim KW, Lee DY, Jhoo JH, Lee SB, Park JH, et al. Development of the Subjective Memory Complaints Questionnaire. Dement Geriatr Cogn Disord 2009;27:310-317.

42. Lee DY, Kim KW, Youn JC, Jhoo JH, Lee JH, Woo JI. Development of an informant report questionnaire for dementia screening: Seoul Informant Report Questionnaire for Dementia (SIRQD). J Korean Neuropsychiatr Assoc 2004;43:209-217.

43. DeCarli C, Mungas D, Harvey D, Reed B, Weiner M, Chui H, et al. Memory impairment, but not cerebrovascular disease, predicts progression of MCI to dementia. Neurology 2004;63:220-227.

44. Laurin D, Masaki KH, White LR, Launer LJ. Ankle-to-brachial index and dementia: the Honolulu-Asia Aging Study. Circulation 2007;116: 2269-2274.

45. Seo EH, Lee DY, Kim KW, Lee JH, Jhoo JH, Youn JC, et al. A normative study of the trail making test in Korean elders. Int J Geriatr Psychiatry 2006;21:844-852.

46. Seo EH, Lee DY, Choo IH, Kim SG, Kim KW, Youn JC, et al. Normative study of the Stroop Color and Word Test in an educationally diverse elderly population. Int J Geriatr Psychiatry 2008;23:1020-1027.

47. Benton AL, Hamsher KD, Sivan AB. Multilingual Aphasia Examination: Manual of Instructions. Iowa City, IA: AJA Assoc; 1994.

48. Wechsler D. WMS-R: Wechsler Memory Scale-Revised: Manual. San Antonio, TX: Psychological Corp., Harcourt Brace Jovanovich; 1987.

49. Choi HJ, Lee DY, Seo EH, Jo MK, Sohn BK, Choe YM, et al. A normative study of the digit span in an educationally diverse elderly population. Psychiatry Investig 2014;11:39-43.

50. Chey J, Kim J, Park KB, Hwang S, Hong S. Korean Wechsler Memory Scale-IV. Daegu: Korea Psychology; 2012.

51. Meyers JE, Meyers KR. Rey Complex Figure Test and Recognition Trial Professional Manual. Odessa, FL: Psychological Assessment Resources; 1995.

52. Hwang S, Kim J, Park K, Chey J, Hong S. Korean Wechsler Adult Intelligence Scale-IV. Daegu: Korea Psychology; 2012.

53. Han JY, Seo EH, Jun JH, Yi D, Sohn BK, Choe YM, et al. Development of the Korean Adult Reading Test (KART) to estimate premorbid intelligence in dementia. Alzheimer Dement 2015;11:P816.

54. Wenham PR, Price WH, Blandell G. Apolipoprotein E genotyping by one-stage PCR. Lancet 1991;337:1158-1159.

55. Buysse DJ, Reynolds CF 3rd, Monk TH, Berman SR, Kupfer DJ. The Pittsburgh Sleep Quality Index: a new instrument for psychiatric prac- 
tice and research. Psychiatry Res 1989;28:193-213.

56. Stiasny-Kolster K, Mayer G, Schäfer S, Möller JC, Heinzel-Gutenbrunner $\mathrm{M}$, Oertel WH. The REM sleep behavior disorder screening questionnaire--a new diagnostic instrument. Mov Disord 2007;22:2386-2393.

57. Chung F, Yegneswaran B, Liao P, Chung SA, Vairavanathan S, Islam S, et al. STOP QuestionnaireA Tool to screen patients for obstructive sleep apnea. J Am Soc Anesthesiol 2008;108:812-821.

58. Do JY, Yoon CH, Kim WJ, Yang KI, Park SH, Chu MK. Prevalence of restless legs syndrome in Korean adult population: comparison between international restless legs syndrome study group criteria and Cambridge-Hopkins diagnostic questionnaire. J Korean Sleep Res Soc 2013;10:7-14.

59. Lee JH, Kim SJ, Lee SY, Jang KH, Kim IS, Duffy JF. Reliability and validity of the Korean version of Morningness-Eveningness Questionnaire in adults aged 20-39 years. Chronobiol Int 2014;31:479-486.

60. Petersen RC, Aisen PS, Beckett LA, Donohue MC, Gamst AC, Harvey DJ, et al. Alzheimer's Disease Neuroimaging Initiative (ADNI): clinical characterization. Neurology 2010;74:201-209.

61. Ellis KA, Bush AI, Darby D, De Fazio D, Foster J, Hudson P, et al. The
Australian Imaging, Biomarkers and Lifestyle (AIBL) study of aging: methodology and baseline characteristics of 1112 individuals recruited for a longitudinal study of Alzheimer's disease. Int Psychogeriatr 2009; 21:672-687.

62. Ossenkoppele R, Jansen WJ, Rabinovici GD, Knol DL, van der Flier WM, van Berckel BN, et al. Prevalence of amyloid PET positivity in dementia syndromes: a meta-analysis. JAMA 2015;313:1939-1949.

63. Rowe CC, Ellis KA, Rimajova M, Bourgeat P, Pike KE, Jones G, et al. Amyloid imaging results from the Australian Imaging, Biomarkers and Lifestyle (AIBL) study of aging. Neurobiol Aging 2010;31:1275-1283.

64. Kye B. Cohort Effects or Period Effects? Fertility Decline in South Korea in the Twentieth Century. Popul Res Policy Rev 2012;31:387-415.

65. Khang YH, Lynch JW, Kaplan GA. Health inequalities in Korea: ageand sex-specific educational differences in the 10 leading causes of death. Int J Epidemiol 2004;33:299-308.

66. Johnson SC, Christian BT, Okonkwo OC, Oh JM, Harding S, Xu G, et al. Amyloid burden and neural function in people at risk for Alzheimer's disease. Neurobiol Aging 2014;35:576-584. 